\title{
Some identities involving second kind Stirling numbers of types $B$ and $D^{*}$
}

\author{
Eli Bagno \\ Jerusalem College of Technology \\ 21 HaVaad HaLeumi St., \\ Jerusalem, Israel \\ bagnoe@g.jct.ac.il
}

\author{
Riccardo Biagioli \\ Institut Camille Jordan, \\ Université Claude Bernard Lyon 1 \\ 69622 Villeurbanne Cedex, France \\ biagioli@math. univ-lyon1.fr
}

\begin{abstract}
David Garber
Department of Applied Mathematics, Holon Institute of Technology,

52 Golomb St., PO Box 305, 58102 Holon, Israel, and (sabbatical:) Einstein Institute of Mathematics, Hebrew University of Jerusalem, Jerusalem, Israel
\end{abstract}

garber@hit.ac.il

Submitted: Apr 30, 2019; Accepted: Jun 20, 2019; Published: Jul 5, 2019

(C) The authors. Released under the CC BY-ND license (International 4.0).

\begin{abstract}
Using Reiner's definition of Stirling numbers of the second kind in types $B$ and $D$, we generalize two well-known identities concerning the classical Stirling numbers of the second kind. The first identity relates them with Eulerian numbers and the second identity interprets them as entries in a transition matrix between the elements of two standard bases of the polynomial ring $\mathbb{R}[x]$. Finally, we generalize these identities to the group of colored permutations $G_{m, n}$.
\end{abstract}

Mathematics Subject Classifications: Primary: 05A18, Secondary: 11B73, $20 \mathrm{~F} 55$

\section{Introduction}

The Stirling number of the second kind, denoted $S(n, k)$, is defined as the number of partitions of the set $[n]:=\{1, \ldots, n\}$ into $k$ non-empty subsets (see [20, page 81]). Stirling numbers of the second kind arise in a variety of problems in enumerative combinatorics;

\footnotetext{
*This research was supported by a grant from the Ministry of Science and Technology, Israel, and the France's Centre National pour la Recherche Scientifique (CNRS)
} 
they have many combinatorial interpretations, and have been generalized in various contexts and in different ways.

In the geometric theory of Coxeter groups they appear as follows. For any finite Coxeter group $W$, there is a corresponding hyperplane arrangement $\mathcal{W}$, whose elements are the reflecting hyperplanes of $W$. Associated with $\mathcal{W}$, there is the set of all the intersections of these hyperplanes, ordered by reverse inclusion, called the intersection lattice, and denoted $L(\mathcal{W})$ (see e.g. [6, 19]). It is well-known that in the Coxeter group of type $A, L\left(\mathcal{A}_{n}\right)$ is isomorphic to the lattice of the set partitions of $[n]$. By this identification, the subspaces of dimension $n-k$ are counted by $S(n, k)$. In this geometric context, Stirling numbers of the second kind are usually called Whitney numbers (see [21, 23] for more details).

For Coxeter groups of types $B$ and $D$, Zaslavsky [23] gave a description of $L\left(\mathcal{B}_{n}\right)$ and $L\left(\mathcal{D}_{n}\right)$ by using the general theory of signed graphs. Then, Reiner [16] gave a different combinatorial representation of $L\left(\mathcal{B}_{n}\right)$ and $L\left(\mathcal{D}_{n}\right)$ in terms of new types of set partitions, called $B_{n^{-}}$and $D_{n}$-partitions. We call the number of $B_{n^{-}}$(resp. $D_{n^{-}}$) partitions with $k$ pairs of nonzero blocks the Stirling number of the second kind of type $B$ (resp. type $D$ ).

The posets of $B_{n^{-}}$and $D_{n}$-partitions, as well as their isomorphic intersection lattices, have been studied in several papers $[4,5,6,10,11,21]$, from algebraic, topological and combinatorial points of view. However, to our knowledge, two famous identities concerning the classical Stirling numbers of the second kind (see e.g. Bona [7, Theorems 1.8 and 1.17]) have not been generalized to types $B$ and $D$ in a combinatorial way: the first identity relates the Stirling numbers to the Eulerian numbers, and the second one formulates a change of bases in $\mathbb{R}[x]$, both will be described below.

The original definition of the Eulerian numbers was given by Euler in an analytic context $[12, \S 13]$. Later, they began to appear in combinatorial problems, as the Eulerian number $A(n, k)$ counts the number of permutations in the symmetric group $S_{n}$ having $k-1$ descents, where a descent of $\sigma \in S_{n}$ is an element of the descent set of $\sigma$, defined by:

$$
\operatorname{Des}(\sigma):=\{i \in[n-1] \mid \sigma(i)>\sigma(i+1)\} .
$$

We denote by $\operatorname{des}(\sigma):=|\operatorname{Des}(\sigma)|$ the descent number.

The first above-mentioned identity relating Stirling numbers of the second kind and Eulerian numbers is the following one, see e.g. [7, Theorem 1.17]:

Theorem 1.1. For all non-negative integers $n \geqslant r$, we have

$$
S(n, r)=\frac{1}{r !} \sum_{k=0}^{r} A(n, k)\left(\begin{array}{l}
n-k \\
r-k
\end{array}\right) .
$$

The second identity arises when one expresses the standard basis of the polynomial ring $\mathbb{R}[x]$ as a linear combination of the basis consisting of the falling factorials (see e.g. the survey of Boyadzhiev [8]):

Theorem 1.2. Let $x \in \mathbb{R}$ and let $n \in \mathbb{N}$. Then we have

$$
x^{n}=\sum_{k=0}^{n} S(n, k)[x]_{k},
$$


where $[x]_{k}:=x(x-1) \cdots(x-k+1)$ is the falling factorial of degree $k$ and $[x]_{0}:=1$.

There are some known proofs for the last identity. A combinatorial one, realizing $x^{n}$ as the number of functions from the set $\{1, \ldots, n\}$ to the set $\{1, \ldots, x\}$ (for a positive integer $x$ ), is presented in [20, Eqn. (1.94d)]. The first geometric proof is due to Knop [13].

In this paper, we use Stirling numbers of the second kind of types $B$ and $D$, in order to generalize the identities stated in Equations (2) and (3). Theorems 4.1 and 4.2 below are generalizations of the first identity for types $B$ and $D$ : they will be proven by providing explicit procedures to construct ordered set partitions starting from the elements of the corresponding Coxeter groups.

Theorems 5.1 and 5.4 generalize the second identity. We present here a geometric approach, suggested to us by Reiner [17], which is based on some geometric characterizations of the intersection lattices of types $B$ and $D$. Moreover, we show how to generalize these two classical identities to the colored permutations group $G_{m, n}$.

The rest of the paper is organized as follows. Sections 2 and 3 present the known generalizations of the Eulerian numbers and the set partitions, respectively, to the Coxeter groups of types $B$ and $D$. In Sections 4 and 5 , we state our generalizations of the two identities and prove them. Finally, in Section 6, we present some possible extensions of the main results.

\section{Eulerian numbers of types $B$ and $D$}

We start with some notations. For $n \in \mathbb{N}$, we set $[ \pm n]:=\{ \pm 1, \ldots, \pm n\}$. For a subset $B \subseteq[ \pm n]$, we denote by $-B$ the set obtained by negating all the elements of $B$, and by $\pm B$ we denote the unordered pair of sets $B,-B$.

Let $(W, S)$ be a Coxeter system. As usual, denote by $\ell(w)$ the length of $w \in W$, which is the minimal integer $k$ satisfying $w=s_{1} \cdots s_{k}$ with $s_{i} \in S$. The (right) descent set of $w \in W$ is defined to be

$$
\operatorname{Des}(w):=\{s \in S \mid \ell(w s)<\ell(w)\} .
$$

A combinatorial characterization of $\operatorname{Des}(w)$ in type $A$ is given by Equation (1) above. Now we recall analogous descriptions in types $B$ and $D$.

We denote by $B_{n}$ the group of all bijections $\beta$ of the set $[ \pm n]$ onto itself such that

$$
\beta(-i)=-\beta(i)
$$

for all $i \in[ \pm n]$, with composition as the group operation. This group is usually known as the group of signed permutations on $[n]$. If $\beta \in B_{n}$, then we write $\beta=[\beta(1), \ldots, \beta(n)]$ and we call this the window notation of $\beta$.

As a set of generators for $B_{n}$ we take $S_{B}:=\left\{s_{0}^{B}, s_{1}^{B}, \ldots, s_{n-1}^{B}\right\}$ where for $i \in[n-1]$

$$
s_{i}^{B}:=[1, \ldots, i-1, i+1, i, i+2, \ldots, n] \text { and } s_{0}^{B}:=[-1,2, \ldots, n] .
$$

It is well-known that $\left(B_{n}, S_{B}\right)$ is a Coxeter system of type $B$ (see e.g. $[3, \S 8.1]$ ). The following characterization of the (right) descent set of $\beta \in B_{n}$ is well-known [3]. 
Proposition 2.1. Let $\beta \in B_{n}$. Then

$$
\operatorname{Des}_{B}(\beta)=\{i \in[0, n-1] \mid \beta(i)>\beta(i+1)\},
$$

where $\beta(0):=0$ (we use the usual order on the integers). In particular, $0 \in \operatorname{Des}_{B}(\beta)$ is a descent if and only if $\beta(1)<0$. We set $\operatorname{des}_{B}(\beta):=\left|\operatorname{Des}_{B}(\beta)\right|$.

For all non-negative integers $n \geqslant k$, we set

$$
A_{B}(n, k):=\left|\left\{\beta \in B_{n} \mid \operatorname{des}_{B}(\beta)=k\right\}\right|,
$$

and we call them the Eulerian numbers of type $B$.

Note that in our context $A_{B}(n, k)$ counts permutations in $B_{n}$ having $k$ descents rather than $k-1$, like in type $A$, since this produces nicer formulas.

We denote by $D_{n}$ the subgroup of $B_{n}$ consisting of all the signed permutations having an even number of negative entries in their window notation. It is usually called the evensigned permutation group. As a set of generators for $D_{n}$ we take $S_{D}:=\left\{s_{0}^{D}, s_{1}^{D}, \ldots, s_{n-1}^{D}\right\}$ where for $i \in[n-1]$ :

$$
s_{i}^{D}:=s_{i}^{B} \text { and } s_{0}^{D}:=[-2,-1,3, \ldots, n] .
$$

It is well-known that $\left(D_{n}, S_{D}\right)$ is a Coxeter system of type $D$, and there is a direct combinatorial way to compute the (right) descent set of $\gamma \in D_{n}$ (see e.g. [3, §8.2]):

Proposition 2.2. Let $\gamma \in D_{n}$. Then

$$
\operatorname{Des}_{D}(\gamma)=\{i \in[0, n-1] \mid \gamma(i)>\gamma(i+1)\},
$$

where $\gamma(0):=-\gamma(2)$. In particular, $0 \in \operatorname{Des}_{D}(\gamma)$ if and only if $\gamma(1)+\gamma(2)<0$. We set $\operatorname{des}_{D}(\gamma):=\left|\operatorname{Des}_{D}(\gamma)\right|$.

For all non-negative integers $n \geqslant k$, we set:

$$
A_{D}(n, k):=\left|\left\{\gamma \in D_{n} \mid \operatorname{des}_{D}(\gamma)=k\right\}\right|,
$$

and we call them the Eulerian numbers of type $D$.

For example, if $\gamma=[1,-3,4,-5,-2,-6]$, then:

$$
\operatorname{Des}_{D}(\gamma)=\{0,1,3,5\}, \text { but } \operatorname{Des}_{B}(\gamma)=\{1,3,5\} .
$$

\section{Set partitions of types $B$ and $D$}

In this section, we introduce the concepts of set partitions of types $B$ and $D$ as defined by Reiner [16].

As mentioned above, we denote by $L(\mathcal{W})$ the intersection lattice corresponding to the Coxeter hyperplane arrangement $\mathcal{W}$ of a finite Coxeter group $W$. We will focus only on 
the hyperplane arrangements of types $A, B$ and $D$. In terms of the coordinate functions $x_{1}, \ldots, x_{n}$ in $\mathbb{R}^{n}$, they can be defined as follows:

$$
\begin{aligned}
& \mathcal{A}_{n}:=\left\{\left\{x_{i}=x_{j}\right\} \mid 1 \leqslant i<j \leqslant n\right\}, \\
& \mathcal{B}_{n}:=\left\{\left\{x_{i}= \pm x_{j}\right\} \mid 1 \leqslant i<j \leqslant n\right\} \cup\left\{\left\{x_{i}=0\right\} \mid 1 \leqslant i \leqslant n\right\}, \\
& \mathcal{D}_{n}:=\left\{\left\{x_{i}= \pm x_{j}\right\} \mid 1 \leqslant i<j \leqslant n\right\} .
\end{aligned}
$$

It is well-known that in type $A$, the intersection lattice $L\left(\mathcal{A}_{n}\right)$ is isomorphic to the lattice of set partitions of $[n]$.

In type $B$, let us consider the following element of $L\left(\mathcal{B}_{9}\right)$ :

$$
\left\{x_{1}=-x_{3}=x_{6}=x_{8}=-x_{9}, x_{2}=x_{4}=0, x_{5}=-x_{7}\right\} \text {. }
$$

It can be easily presented as the following set partition of $[ \pm 9]$ :

$$
\{\{1,-3,6,8,-9\},\{-1,3,-6,-8,9\},\{2,-2,4,-4\},\{5,-7\},\{-5,7\}\} .
$$

This probably was Reiner's motivation to define the set partitions of type $B$, as follows:

Definition 3.1. A $B_{n}$-partition is a set partition of $[ \pm n]$ into blocks such that the following conditions are satisfied:

- There exists at most one block satisfying $-C=C$, called the zero-block. It is a subset of $[ \pm n]$ of the form $\{ \pm i \mid i \in S\}$ for some $S \subseteq[n]$.

- If $C$ appears as a block in the partition, then $-C$ also appears in that partition.

A similar definition holds for set partitions of type $D$ :

Definition 1. A $D_{n}$-partition is a $B_{n}$-partition such that the zero-block, if exists, contains at least two positive elements.

We denote by $S_{B}(n, r)$ (resp. $\left.S_{D}(n, r)\right)$ the number of $B_{n^{-}}\left(\right.$resp. $\left.D_{n^{-}}\right)$partitions having exactly $r$ pairs of nonzero blocks. These numbers are called Stirling numbers (of the second kind) of type $B$ (resp. type $D$ ). They correspond, respectively, to the sequences oeis.org/A039755 and oeis.org/A039760 in the OEIS. Tables 1 and 2 record these numbers for small values of $n$ and $r$.

We now define the concept of an ordered set partition:

Definition 3.2. A $B_{n}$-partition (or $D_{n}$-partition) is ordered if the set of blocks is totally ordered and the following conditions are satisfied:

- If the zero-block exists, then it appears as the first block.

- For each block $C$ which is not the zero-block, the blocks $C$ and $-C$ are adjacent.

Example 3.3. The following partitions

$$
\begin{aligned}
& P_{1}=\{\{ \pm 3\}, \pm\{-2,1\}, \pm\{-4,5\}\}, \\
& P_{2}=\{ \pm\{1\}, \pm\{2\}, \pm\{-4,3\}\}, \\
& P_{3}=[\{ \pm 1, \pm 3\},\{-2\},\{2\},\{-4,5\},\{-5,4\}],
\end{aligned}
$$

are respectively, a $B_{5}$-partition which is not a $D_{5}$-partition, a $D_{4}$-partition with no zeroblock, and an ordered $D_{5}$-partition having a zero-block. 


\begin{tabular}{r||r|r|r|r|r|r|r}
$n / r$ & 0 & 1 & 2 & 3 & 4 & 5 & 6 \\
\hline \hline 0 & 1 & & & & & & \\
1 & 1 & 1 & & & & & \\
2 & 1 & 4 & 1 & & & & \\
3 & 1 & 13 & 9 & 1 & & & \\
4 & 1 & 40 & 58 & 16 & 1 & & \\
5 & 1 & 121 & 330 & 170 & 25 & 1 & \\
6 & 1 & 364 & 1771 & 1520 & 395 & 36 & 1
\end{tabular}

Table 1: Stirling numbers $S_{B}(n, r)$ of the second kind of type $B$.

\begin{tabular}{r||r|r|r|r|r|r|r}
$n / r$ & 0 & 1 & 2 & 3 & 4 & 5 & 6 \\
\hline \hline 0 & 1 & & & & & & \\
1 & 0 & 1 & & & & & \\
2 & 1 & 2 & 1 & & & & \\
3 & 1 & 7 & 6 & 1 & & & \\
4 & 1 & 24 & 34 & 12 & 1 & & \\
5 & 1 & 81 & 190 & 110 & 20 & 1 & \\
6 & 1 & 268 & 1051 & 920 & 275 & 30 & 1
\end{tabular}

Table 2: Stirling numbers $S_{D}(n, r)$ of the second kind of type $D$.

\section{Connections between Stirling and Eulerian numbers of types $B$ and $D$}

In this section, we present two generalizations of Theorem 1.1 for Coxeter groups of types $B$ and $D$.

Theorem 4.1. For all non-negative integers $n \geqslant r$, we have:

$$
S_{B}(n, r)=\frac{1}{2^{r} r !} \sum_{k=0}^{r} A_{B}(n, k)\left(\begin{array}{l}
n-k \\
r-k
\end{array}\right) .
$$

Theorem 4.2. For all non-negative integers $n \geqslant r$, with $n \neq 1$, we have:

$$
S_{D}(n, r)=\frac{1}{2^{r} r !}\left[\sum_{k=0}^{r} A_{D}(n, k)\left(\begin{array}{l}
n-k \\
r-k
\end{array}\right)+n \cdot 2^{n-1}(r-1) ! \cdot S(n-1, r-1)\right],
$$

where $S(n-1, r-1)$ is the usual Stirling number of the second kind.

Now, by inverting these formulas, similarly to the known equality in type $A$, mentioned in [7, Corollary 1.18]:

$$
A(n, k)=\sum_{r=1}^{k}(-1)^{k-r} \cdot r ! \cdot S(n, r) \cdot\left(\begin{array}{c}
n-r \\
k-r
\end{array}\right)
$$


we get the following expressions for the Eulerian numbers of type $B$ (resp. type $D$ ) in terms of the Stirling numbers of type $B$ (resp. type $D)$ :

Corollary 4.3. For all non-negative integers $n \geqslant k$, we have:

$$
A_{B}(n, k)=\sum_{r=0}^{k}(-1)^{k-r} \cdot 2^{r} r ! \cdot S_{B}(n, r) \cdot\left(\begin{array}{c}
n-r \\
k-r
\end{array}\right) .
$$

Corollary 4.4. For all non-negative integers $n \geqslant k$, with $n \neq 1$, we have:

$$
A_{D}(n, k)=\left[\sum_{r=0}^{k}(-1)^{k-r} \cdot 2^{r} r ! \cdot S_{D}(n, r) \cdot\left(\begin{array}{c}
n-r \\
k-r
\end{array}\right)\right]-n \cdot 2^{n-1} \cdot A(n-1, k-1) .
$$

A different equality for $A_{D}(n, k)$ can be found in Brenti [9, Thm. 4.13].

\subsection{Proof for type $B$}

The proofs in this and in the next subsections use arguments similar to Bona's proof for the corresponding identity for type $A$, see [7, Theorem 1.17].

Proof of Theorem 4.1. We have to prove the following equality:

$$
2^{r} r ! S_{B}(n, r)=\sum_{k=0}^{r} A_{B}(n, k)\left(\begin{array}{l}
n-k \\
r-k
\end{array}\right) .
$$

The number $2^{r} r ! S_{B}(n, r)$ in the left-hand side is the number of ordered $B_{n}$-partitions having $r$ pairs of nonzero blocks. Now, let us show that the right-hand side counts the same set of partitions in a different way.

Let $\beta \in B_{n}$ be a signed permutation with $\operatorname{des}_{B}(\beta)=k$, written in its window notation. We start by adding a separator after each descent of $\beta$ and after $\beta(n)$. If $0 \in \operatorname{Des}_{B}(\beta)$, this means that a separator is added before $\beta(1)$. If $r>k$, we add extra $r-k$ artificial separators in some of the $n-k$ empty spots, where by a spot we mean a gap between two consecutive entries of $\beta$ or the gap before the first entry $\beta(1)$. This splits $\beta$ into a set of $r$ blocks, where the block $C_{i}$ is defined as the set of entries between the $i$ th and the $(i+1)$ th separators for $1 \leqslant i \leqslant r$. Now, this set of blocks is transformed into the ordered $B_{n}$-partition with $r$ pairs of nonzero blocks:

$$
\left[C_{0}, C_{1},-C_{1}, \ldots, C_{r},-C_{r}\right]
$$

where the (optional) zero-block $C_{0}$ equals to $\{ \pm \beta(1), \ldots, \pm \beta(j)\}$ if the first separator is after $\beta(j)$, for some $j \geqslant 1$, and it does not exist if the first separator is before $\beta(1)$.

For example, if $\beta=[-2,3,5,1,-4] \in B_{5}$, then after adding the separators induced by descents, we get the sequence $[|-2,3,5| 1|-4|]$, which is transformed into the ordered partition $[\{-2,3,5\},\{2,-3,-5\},\{1\},\{-1\},\{-4\},\{4\}]$. On the other hand, if $\beta^{\prime}=[2,3,5,-1,-4] \in B_{5}$, then after adding the separators induced by the descents, we have $\beta^{\prime}=[2,3,5|-1|-4 \mid]$, which gives rise to the ordered partition $[\{ \pm 2, \pm 3, \pm 5\},\{-1\},\{1\},\{-4\},\{4\}]$, with a zero-block, and two nonzero blocks. 
There are exactly $\left(\begin{array}{c}n-k \\ r-k\end{array}\right)$ ordered $B_{n}$-partitions obtained from $\beta$ in this way. From now on, we refer to this process of creating $B_{n}$-partitions starting from a single signed permutation $\beta$, as the $B$-procedure.

It is easy to see that the $B$-procedure applied to different signed permutations produces disjoint sets of ordered $B_{n}$-partitions; therefore, one can create in this way

$$
\sum_{k=0}^{r} A_{B}(n, k)\left(\begin{array}{l}
n-k \\
r-k
\end{array}\right)
$$

distinct ordered $B_{n}$-partitions with $r$ pairs of nonzero blocks.

Let us show that any ordered $B_{n}$-partition $\lambda=\left[C_{0}, C_{1},-C_{1}, \ldots, C_{r},-C_{r}\right]$, can be obtained through the $B$-procedure. If $\lambda$ contains a zero-block $C_{0}$, then put the positive elements of $C_{0}$ in increasing order at the beginning of a sequence $\mathbf{S}$, and add a separator after them. Then, order increasingly the elements in each of the blocks $C_{1}, \ldots, C_{r}$, and write them sequentially in $\mathbf{S}$ (after the first separator if exists), by adding a separator after the last entry coming from each block. Reading the formed sequence $\mathbf{S}$ from the left to the right, one obtains the window notation of a signed permutation $\beta$. Note that the number of descents in $\beta$ is smaller than or equal to $r$, since the elements in each block are ordered increasingly. Now, it is clear that $\lambda$ can be obtained by applying the $B$-procedure to $\beta$, where the artificial separators are easily recovered.

Example 4.5. The signed permutation

$$
\beta=[1,4|-5,-3,2|] \in B_{5}
$$

has 2 as a descent. It produces the following ordered $B_{5}$-partition with one pair of nonzero blocks

$$
[\{ \pm 1, \pm 4\},\{-5,-3,2\},\{5,3,-2\}]
$$

and exactly $\left(\begin{array}{l}4 \\ 1\end{array}\right)$ ordered $B_{5}$-partitions with two pairs of nonzero blocks, namely:

$$
\begin{gathered}
{[\{1,4\},\{-1,-4\},\{-5,-3,2\},\{5,3,-2\}],} \\
{[\{ \pm 1\},\{4\},\{-4\},\{-5,-3,2\},\{5,3,-2\}],} \\
{[\{ \pm 1, \pm 4\},\{-5\},\{5\},\{-3,2\},\{3,-2\}],} \\
{[\{ \pm 1, \pm 4\},\{-5,-3\},\{5,3\},\{2\},\{-2\}],}
\end{gathered}
$$

obtained by placing one artificial separator before entries 1,2,4 and 5, respectively. The other ordered partitions coming from $\beta$ with more blocks are obtained similarly.

Conversely, let

$$
\lambda=[\{ \pm 1, \pm 4\},\{5\},\{-5\},\{-3,2\},\{3,-2\}]
$$

be an ordered $B_{5}$-partition. The corresponding signed permutation with the added separators is $\beta=[1,4 \| 5|-3,2|] \in B_{5}$. Note that although $C_{1}=\{5\}$ is a separate block, there is no descent between 4 and 5 , meaning that $\lambda$ is obtained by adding an artificial separator in the spot between these two entries, denoted $\|$. 


\subsection{Proof for type $D$}

The proof of Theorem 4.2 is a bit more tricky. The basic idea is the same as before: obtaining the whole set of ordered $D_{n}$-partitions with $r$ pairs of nonzero blocks from elements in $D_{n}$ with at most $r$ descents. We will use the $B$-procedure presented in the previous subsection, with the addition of an extra step, to take care of the special structure of the $D_{n}$-partitions. First of all, we recall that we might have $\operatorname{Des}_{D}(\gamma) \neq \operatorname{Des}_{B}(\gamma)$ for $\gamma \in D_{n}$, see an example at the end of Section 2 .

Let $\gamma \in D_{n}$ be such that $\operatorname{des}_{D}(\gamma)=k$. We start by adding the separators after the $D$-descents of $\gamma$ and the artificial ones in case that $k<r$. Using the $B$-procedure, we transform $\gamma$, equipped with the set of separators, into a $B_{n}$-partition. The result is also a $D_{n}$-partition, except in the case when there is a separator (either induced by a $D$-descent or by an artificial addition) between $\gamma(1)$ and $\gamma(2)$, but not before $\gamma(1)$. In fact, in this case, we obtain an ordered $B_{n}$-partition with a zero-block containing exactly one pair of elements, which is not a $D_{n}$-partition.

Hence, only in this case, we slightly modify the procedure as follows. First we toggle the sign of $\gamma(1)$ and move the separator from after $\gamma(1)$ to before it. We call this action the switch operation. Then, we transform this new sequence of entries and separators into a $D_{n}$-partition by applying the $B$-procedure. We refer to this process of associating a permutation $\gamma \in D_{n}$ with the obtained set of ordered $D_{n}$-partitions, as the $D$-procedure.

Before proving that this procedure indeed creates ordered $D_{n}$-partitions, we give an example of an element $\gamma \in D_{n}$, for which the application of the switch operation is required.

Example 4.6. Let $\gamma=[-1|| 3,4|-2|-6,-5 \mid] \in D_{6}$ be a permutation equipped with the separators induced by the $D$-descents and one artificial separator added after position 1. The $B$-procedure, applied to $\gamma$, results in an illegal ordered $D_{6}$-partition, since the zero-block $B_{0}=\{ \pm 1\}$ consists of only one pair. Toggling the sign of $\gamma(1)$, and moving the artificial separator before position 1, we obtain:

$$
\gamma^{\prime}=[|| 1,3,4|-2|-6,-5 \mid] \in B_{6} \backslash D_{6},
$$

that is transformed into the ordered $D_{6}$-partition:

$$
[\{1,3,4\},\{-1,-3,-4\},\{-2\},\{2\},\{-6,-5\},\{6,5\}] .
$$

As in type $B$, it is easy to see that by applying the $D$-procedure to all the permutations in $D_{n}$, we obtain disjoint sets of ordered $D_{n}$-partitions, though, in this case we do not obtain all of them. The next lemma specifies exactly which $D_{n}$-partitions are not reached, but first we need to define the notions of odd and even partitions:

Definition 2. An ordered $D_{n}$-partition $\lambda=\left[C_{0}, C_{1},-C_{1}, \ldots, C_{k},-C_{k}\right]$ is called odd if the number of negative elements in $C_{1} \cup C_{2} \cup \cdots \cup C_{k}$ is odd, and otherwise it will be called even.

Then, we have the following: 
Lemma 4.7. The ordered $D_{n}$-partitions with $r$ pairs of nonzero blocks, which cannot be obtained by the $D$-procedure are exactly the odd $D_{n}$-partitions of of the form

$$
\lambda=\left[C_{1}=\{*\},-C_{1}=-\{*\}, C_{2},-C_{2}, \ldots, C_{r},-C_{r}\right],
$$

where $*$ stands for a single element of $[ \pm n]$.

Proof. First of all, we remark that after the $D$-procedure is applied to a permutation (equipped with separators) with the use of the switch operation, only $D_{n}$-partitions of the form $\left[C_{1},-C_{1}, \ldots, C_{r},-C_{r}\right]$ without a zero-block and $\left|C_{1}\right| \geqslant 2$ can be obtained.

Now, we move to permutations in $D_{n}$ which the $D$-procedure is applied without the use of the switch operation. We have one of the following possibilities with regard to the separators before and after position 1 :

- There are no separators before and after position 1: this means that $\left|C_{0}\right| \geqslant 2$.

- There is a separator before position 1 but not after it: this means that $\left|C_{1}\right| \geqslant 2$ and $C_{0}=\emptyset$.

- There are two separators before and after position 1: this means that $\left|C_{1}\right|=1$ and $C_{0}=\emptyset$. Note that in this case, by the $D$-procedure, we get an ordered $D_{n^{-}}$ partition $\left[C_{1},-C_{1}, \ldots, C_{r},-C_{r}\right]$ with an even number of negative entries in the union $C_{1} \cup C_{2} \cup \cdots \cup C_{r}$.

From all the above, it follows that the partitions in the formulation of the lemma cannot be reached.

Conversely, let us show that all other ordered $D_{n}$-partitions can be obtained using the $D$-procedure.

Let $\lambda=\left[C_{0}, C_{1},-C_{1}, \ldots, C_{r},-C_{r}\right]$ be an ordered $D_{n}$-partition with $C_{0} \neq \emptyset$, and so $C_{0}$ consists of at least two pairs of elements. We look for the preimage $\gamma \in D_{n}$ of $\lambda$. Since the switch operation on a permutation $\gamma \in D_{n}$ produces $D_{n}$-partitions without a zero-block, in our case the switch operation has not been applied to $\gamma$.

We start by defining a sequence $\mathbf{S}$ as follows: we first put the positive entries of $C_{0}$ in their natural increasing order as the first elements of $\mathbf{S}$, followed by a separator. If $\lambda$ is odd, we change the sign of the first entry of $\mathbf{S}$ to be negative. Now, as described in the proof of Theorem 4.1, we complete $\mathbf{S}$ by concatenating the $r$ sequences created by the elements of the blocks $C_{1}, \ldots, C_{r}$, where in each block the elements are ordered increasingly and followed by a separator. We now consider the obtained sequence $\mathbf{S}$ as a permutation $\gamma \in D_{n}$. Note that $0 \notin \operatorname{Des}_{D}(\gamma)$, since by construction $|\gamma(1)|<\gamma(2)$ and so $\gamma(1)+\gamma(2)>0$. Moreover, it is clear that applying the $D$-procedure to $\gamma$ yields the $D_{n}$-partition $\lambda$ (see e.g. Example 4.8(a)).

Now assume that $\lambda=\left[C_{1},-C_{1}, \ldots, C_{r},-C_{r}\right]$ is an ordered $D_{n}$-partition with no zeroblock.

If $\lambda$ is even, it is easy to see that the above construction without the initial step of reordering the zero-block $C_{0}$ produces $\gamma \in D_{n}$ which is the preimage of $\lambda$. 
Finally, if $\lambda=\left[C_{1},-C_{1}, \ldots, C_{r},-C_{r}\right]$ is odd and is not listed in Equation (7), it means that $\left|C_{1}\right| \geqslant 2$. As before, we define a sequence $\mathbf{S}$ by reordering increasingly all the blocks $C_{i}$. Since $\left|C_{1}\right| \geqslant 2$, we have by the construction that $\mathbf{S}(1)<\mathbf{S}(2)$. Since the partition is odd with no zero-block, we have applied a switch operation on its preimage. Now consider $\mathbf{S}$ as a permutation $\gamma \in D_{n}$. It is easy to see that the obtained permutation $\gamma \in D_{n}$ is indeed the preimage of $\lambda$ (see e.g. Example 4.8(b)).

We give now two examples of the reverse procedure: both examples are ordered odd $D_{5}$-partitions, but one has a zero-block, while the other has no zero-block, so the latter requires the switch operation.

Example 4.8. (a) Let

$$
\lambda_{1}=\left[C_{0}=\{ \pm 1, \pm 4\},\{3\},\{-3\},\{-5,2\},\{5,-2\}\right]
$$

be an odd ordered $D_{5}$-partition with a zero-block $C_{0}$ which is odd since we have one negative sign in $\{3\} \cup\{-5,2\}$. For recovering its preimage $\gamma_{1} \in D_{5}$, we choose the negative sign for the smallest positive entry in the zero-block, which is 1. After inserting the other positive entry of $C_{0}$ and a separator, we insert the other blocks, where each block is ordered increasingly followed by a separator, to obtain the permutation:

$$
\gamma_{1}=[-1,4|3|-5,2 \mid] \in D_{5},
$$

which is the preimage of the partition $\lambda_{1}$ using the $D$-procedure.

(b) Let

$$
\lambda_{2}=[\{2,3\},\{-2,-3\},\{-1\},\{1\}]
$$

be an ordered odd $D_{3}$-partition without a zero-block. Hence, it is created by the switch operation. First, by the standard reverse procedure, we get the element:

$$
\gamma_{2}^{\prime}=[|2,3|-1 \mid] \in B_{3} \backslash D_{3} .
$$

Then, after performing the toggling of the sign of the first digit induced by the switch, we obtain:

$$
\gamma_{2}=[-2 \| 3 \mid 1] \in D_{3},
$$

that is the permutation from which the partition $\lambda_{2}$ is obtained.

We can now complete the proof of Theorem 4.2 .

Proof of Theorem 4.2. The equation in the statement of the theorem is equivalent to the following:

$$
2^{r} r ! S_{D}(n, r)=\sum_{k=0}^{r} A_{D}(n, k)\left(\begin{array}{c}
n-k \\
r-k
\end{array}\right)+n \cdot 2^{n-1}(r-1) ! \cdot S(n-1, r-1) .
$$

The left-hand side of the above equation counts the number of ordered $D_{n}$-partitions with $r$ pairs of nonzero blocks. The right-hand side counts the same set of partitions 
divided in two categories: those coming from the $D$-procedure, that are induced by permutations counted in $A_{D}(n, k)$, and those that are not, which are listed in Lemma 4.7. It is easy to see that the latter can be enumerated in the following way: first choose the absolute value of the unique element in $C_{1}=\{*\}$, which can be done in $n$ ways. Then, choose and order the $r-1$ remaining blocks, which can be done in $(r-1) ! \cdot S(n-1, r-1)$ ways. Finally, choose the sign of each entry in the blocks $C_{1}, C_{2}, \ldots, C_{r}$, in such a way that an odd number of entries will be signed, and this can be done in $2^{n-1}$ ways. This completes the proof.

\section{$5 \quad$ Falling factorials for Coxeter groups of types $B$ and $D$}

In this section, we present generalizations of Theorem 1.2 for Coxeter groups of types $B$ and $D$ and provide combinatorial proofs for them.

\section{$5.1 \quad$ Type $B$}

The following theorem is a natural generalization of Theorem 1.2 for the Stirling numbers of type $B$, and it is a particular case of an identity appearing in Remmel and Wachs [18] (specifically, this is $S_{n, k}^{0,2}(1,1)$ in their notation) and Bala [2] (specifically, this is $S_{(2,0,1)}$ in his notation):

Theorem 5.1. Let $x \in \mathbb{R}$ and let $n \in \mathbb{N}$. Then we have

$$
x^{n}=\sum_{k=0}^{n} S_{B}(n, k)[x]_{k}^{B},
$$

where $[x]_{k}^{B}:=(x-1)(x-3) \cdots(x-2 k+1)$ and $[x]_{0}^{B}:=1$.

A proof for this equality, using the combinatorial interpretation of $S_{B}(n, k)$ by the model of $k$-attacking rooks, is given by Remmel and Wachs [18]. More information on the rook interpretation of this and other factorization theorems can be found in Miceli and Remmel [14].

Bala [2] uses generating functions techniques for proving this identity.

Here we provide a kind of a geometric proof, suggested to us by Reiner, which is related to a method used by Blass and Sagan [6] to compute the characteristic polynomial of the poset $L\left(\mathcal{B}_{n}\right)$.

Proof. Being a polynomial identity, it is sufficient to prove it only for odd integers $x=$ $2 m+1$ where $m \in \mathbb{N}$.

The left-hand side of Equation (8) counts the number of lattice points in the $n$ dimensional cube $\{-m,-m+1, \ldots,-1,0,1, \ldots, m\}^{n}$. We show that the right-hand side of Equation (8) counts the same set of points using the maximal intersection subsets of hyperplanes the points lay on.

More precisely, let $\lambda=\left\{C_{0}, \pm C_{1}, \ldots, \pm C_{k}\right\}$ be a $B_{n}$-partition with $k$ pairs of nonzero blocks, with $0 \leqslant k \leqslant n$. We associate to this partition the set of lattice points of the 
form $\left(x_{1}, \ldots, x_{n}\right)$, where $x_{j}=0$ for all $j \in C_{0}$, and $x_{j_{1}}=x_{j_{2}} \neq 0\left(\right.$ resp. $\left.x_{j_{1}}=-x_{j_{2}} \neq 0\right)$ whenever $j_{1}, j_{2}$ (resp. $\left.j_{1},-j_{2}\right)$ belong to the same block $C_{i}$ (resp. $-C_{i}$ ).

For the first pair of nonzero blocks $\pm C_{1}$ of the set partition $\lambda$, if $j_{1} \in C_{1} \cup-C_{1}$ then there are $x-1$ possibilities (excluding the value 0 ) to choose the value of $x_{j_{1}}$. For the second pair of blocks $\pm C_{2}$ of the partition $\lambda$, we have $x-3$ possibilities (excluding the value 0 and the value $x_{j_{1}}$ chosen for $\pm C_{1}$ and its negative). We continue in this way until we get $x-(2 k-3)$ possibilities for the last pair of blocks $\pm C_{k}$.

In particular, for $k=0, \lambda$ consists of only the zero-block $\{ \pm 1, \ldots, \pm n\}$, and is associated with the single lattice point $(0, \ldots, 0)$; for $k=n$, the only $B_{n}$-partition having $n$ pairs of nonzero blocks is

$$
\{ \pm\{1\}, \ldots, \pm\{n\}\}
$$

which corresponds to the lattice points $\left(x_{1}, \ldots, x_{n}\right)$ such that $x_{i} \neq \pm x_{j} \neq 0$ for all $i \neq j$. Note that these are the $(x-1)(x-3) \cdots(x-(2 n-1))$ lattice points that do not lie on any hyperplane.

Example 5.2. Let $n=2$ and $m=3$, so we have that $x=2 m+1=7$. The lattice $([-3,3] \times[-3,3]) \cap \mathbb{Z}^{2}$ is presented in Figure 1 .

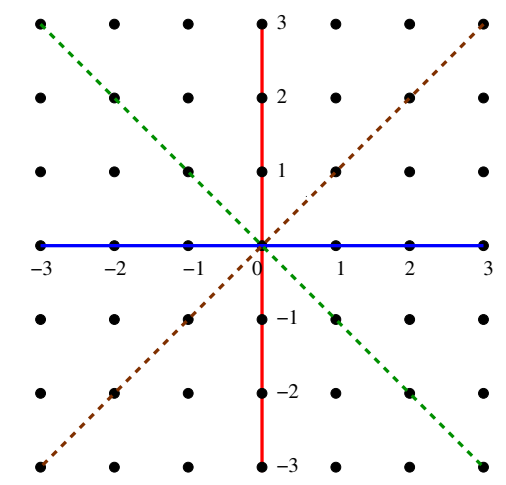

Figure 1: Lattice points for type $B$

For $k=0$, we have exactly one $B_{2}$-partition $\lambda_{0}$ consisting only of the zero-block: $\lambda_{0}=\{\{ \pm 1, \pm 2\}\}$. The corresponding subspace is $\left\{x_{1}=x_{2}=0\right\}$, which counts only the lattice point $(0,0)$.

For $k=1$, we have four $B_{2}$-partitions, two of them contain a zero-block:

$$
\lambda_{1}=\{\{ \pm 1\}, \pm\{2\}\} ; \quad \lambda_{2}=\{\{ \pm 2\}, \pm\{1\}\}
$$

and two of them do not:

$$
\lambda_{3}=\{ \pm\{1,2\}\} ; \quad \lambda_{4}=\{ \pm\{1,-2\}\} .
$$

The partitions $\lambda_{1}$ and $\lambda_{2}$ correspond to the axes $x_{1}=0$ and $x_{2}=0$, respectively. The second pair $\lambda_{3}$ and $\lambda_{4}$ corresponds to the diagonals $x_{1}=x_{2}$ and $x_{1}=-x_{2}$ respectively. Each of these hyperplanes contains 6 lattice points (since the origin is excluded). 
For $k=2$, the single $B_{2}$-partition:

$$
\lambda_{5}=\{ \pm\{1\}, \pm\{2\}\}
$$

corresponds to the set of lattice points $\left(x_{1}, x_{2}\right)$ with $x_{1} \neq \pm x_{2} \neq 0$, which are those not lying on any hyperplane.

Remark 5.3. Note that Blass and Sagan [6, Theorem 2.1] show that, when $x$ is an odd number, the cardinality of the set of lattice points not lying on any hyperplane is counted by the characteristic polynomial $\chi\left(\mathcal{B}_{n}, x\right)$ of the lattice $L\left(\mathcal{B}_{n}\right)$.

\subsection{Type $D$}

The falling factorial in type $D$ is defined as follows: (see [6])

$$
[x]_{k}^{D}:= \begin{cases}1, & k=0 ; \\ (x-1)(x-3) \cdots(x-(2 k-1)), & 1 \leqslant k<n ; \\ (x-1)(x-3) \cdots(x-(2 n-3))(x-(n-1)), & k=n .\end{cases}
$$

We have found no generalization of Equation (3) for type $D$ in the literature, so we supply one here.

Theorem 5.4. For all $n \in \mathbb{N}$ and $x \in \mathbb{R}$ :

$$
x^{n}=\sum_{k=0}^{n} S_{D}(n, k)[x]_{k}^{D}+n\left((x-1)^{n-1}-[x]_{n-1}^{D}\right) .
$$

Proof. For $D_{n}$-partitions having $0 \leqslant k<n$ pairs of nonzero blocks the proof goes verbatim as in type $B$, so let $k=n$.

In this case, we have only one possible $D_{n}$-partition having $n$ pairs of nonzero blocks: $\{ \pm\{1\}, \ldots, \pm\{n\}\}$. We associate this $D_{n}$-partition with the lattice points of the form $\left(x_{1}, \ldots, x_{n}\right)$ such that $x_{i} \neq \pm x_{j}$ for $i \neq j$, having at most one appearance of the value 0 . Note that the points with exactly one appearance of 0 cannot be obtained by any $D_{n}$-partition having $k<n$ blocks, since the zero-block cannot consist of exactly one pair. If 0 does appear, then we have to place it in one of the $n$ coordinates and then we are left with $(x-1)(x-3) \cdots(x-(2 n-3))$ possibilities for the rest, while if 0 does not exist, then we have $(x-1)(x-3) \cdots(x-(2 n-1))$ possibilities. These two values sum up to a total of

$$
[x]_{n}^{D}=(x-1)(x-3) \cdots(x-(2 n-3))(x-(n-1)) .
$$

As in type $B$, this number is equal to the evaluation of the characteristic polynomial $\chi\left(\mathcal{D}_{n}, x\right)$ of $L\left(\mathcal{D}_{n}\right)$, where $x$ is odd.

Note that during the above process of collecting lattice points of the $n$-dimensional cube, the points containing exactly one appearance of 0 and at least two nonzero coordinates are assigned the same absolute value are not counted, since the zero-block (if exists) must contain at least two elements. This phenomenon happens when $n>2$, and the number of such points is $n\left((x-1)^{n-1}-[x]_{n-1}^{D}\right)$. This concludes the proof. 


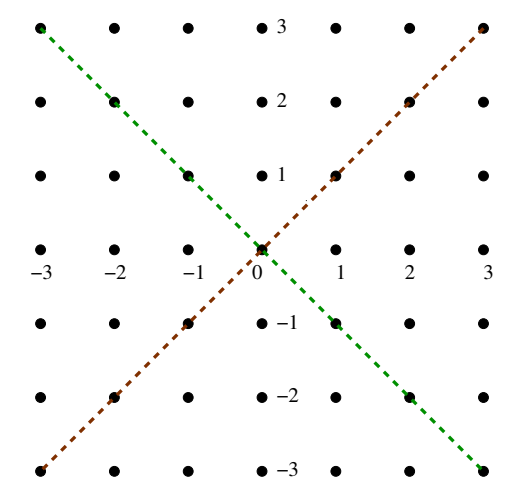

Figure 2: Lattice points for type $D$

Example 5.5. As in the previous example, let $n=2$ and $m=3$, so we have: $x=$ $2 m+1=7$. The lattice $([-3,3] \times[-3,3]) \cap \mathbb{Z}^{2}$ is presented in Figure 2 .

For $k=0$, as in type $B$ we have exactly one $D_{2}$-partition $\lambda_{0}=\{\{ \pm 1, \pm 2\}\}$ which counts only the lattice point $(0,0)$. For $k=1$, we have only two $D_{2}$-partitions: $\{ \pm\{1,2\}\}$ and $\{ \pm\{1,-2\}\}$, which correspond, as in the previous example, to the diagonals $x_{1}=x_{2}$ and $x_{1}=-x_{2}$ (without the origin), respectively

For $k=2$, as before, there is a single $D_{n}$-partition with two pairs of nonzero blocks: $\lambda=\{ \pm\{1\}, \pm\{2\}\}$. The lattice points corresponding to this set partition are those with different values in their coordinates, i.e. $x_{1} \neq x_{2}$, but in the case of type $D$ (in contrast to type $B$ ) the value 0 can also appear. In the figure, these are all the lattice points which do not lie on the diagonals.

Note that in the case $n=2$ the second term in Equation (9) is 0 and hence does not count any missing lattice points, since we have already counted all the points. The missing points start to appear from $n=3$, as presented in the next example.

Example 5.6. Let $n=m=3$, so that $x=2 m+1=7$. The lattice points which are not counted have the form $\left(x_{1}, x_{2}, x_{3}\right)$, such that exactly one of their coordinates is 0 and the other two share the same absolute value, e.g. the lattice points $(0,2,2)$ and $(0,2,-2)$ are not counted. In this case, the number of such missing lattice points (which is the second summand in the right-hand side of Equation (9)) is: $3 \cdot 6^{2}-3 \cdot 6 \cdot 4=36$.

\section{Some generalizations}

In this section, we present some generalizations and variants related to our main results in some different directions. In Section 6.1, we start with a short introduction to the colored permutations group and we generalize Theorems 4.1 and 5.1 to this case. In Section 6.2, we provide a version of Theorem 4.1 for the flag descent parameter in type $B$. 


\subsection{The colored permutations group}

Definition 6.1. Let $m$ and $n$ be positive integers. The group of colored permutations of $n$ digits with $m$ colors is the wreath product

$$
G_{m, n}=\mathbb{Z}_{m} \curlywedge S_{n}=\mathbb{Z}_{m}^{n} \rtimes S_{n},
$$

consisting of all the pairs $(\vec{z}, \tau)$, where $\vec{z}$ is an $n$-tuple of integers between 0 and $m-1$ and $\tau \in S_{n}$.

A convenient way to look at $G_{m, n}$ is to consider the alphabet

$$
\Sigma:=\left\{1, \ldots, n, 1^{[1]}, \ldots, n^{[1]}, \ldots, 1^{[m-1]}, \ldots, n^{[m-1]}\right\}
$$

as the set $[n]$ colored by the colors $0, \ldots, m-1$. Then, an element of $G_{m, n}$ is a bijection $\pi: \Sigma \rightarrow \Sigma$ satisfying the following condition:

$$
\text { if } \pi\left(i^{[\alpha]}\right)=j^{[\beta]} \text {, then } \pi\left(i^{[\alpha+1]}\right)=j^{[\beta+1]},
$$

where the exponent [.] is computed modulo $m$. The elements of $G_{m, n}$ are usually called colored permutations.

In particular, $G_{1, n}=S_{n}$ is the symmetric group, while $G_{2, n}=B_{n}$ is the group of signed permutations.

Definition 6.2. The color order on $\Sigma$ is defined to be (see $[1, \S 3])$ :

$$
1^{[m-1]} \prec \cdots \prec n^{[m-1]} \prec \cdots \prec 1^{[1]} \prec 2^{[1]} \prec \cdots \prec n^{[1]} \prec 1 \prec \cdots \prec n .
$$

Definition 6.3. Let $\pi \in G_{m, n}$. Assume that $\pi(1)=a_{1}^{\left[z_{1}\right]}$. We define

$$
\operatorname{des}_{G}(\pi):=\operatorname{des}_{A}(\pi)+\varepsilon(\pi),
$$

where

$$
\operatorname{des}_{A}(\pi):=|\{i \in[n-1] \mid \pi(i) \succ \pi(i+1)\}|,
$$

where ' $\succ$ ' refers to the color order, and

$$
\varepsilon(\pi):= \begin{cases}1, & \text { if } z_{1} \neq \equiv 0 \bmod m \\ 0, & \text { if } z_{1} \equiv 0 \bmod m\end{cases}
$$

For example, if $\pi=[3, \overline{1}, \overline{\overline{2}}] \in G_{3,3}$, we have $\operatorname{des}_{G}(\pi)=2+0=2$. Note that for $m=1$, $\operatorname{des}_{G}=\operatorname{des}$ and for $m=2, \operatorname{des}_{G}=\operatorname{des}_{B}$.

Moreover, we define the Eulerian number of type $G_{m, n}$ to be:

$$
A_{m}(n, k):=\left|\left\{\pi \in G_{m, n} \mid \operatorname{des}_{G}(\pi)=k\right\}\right| .
$$

Let $C \subseteq \Sigma$. Denote $C^{[t]}=\left\{x^{[i+t]} \mid x^{[i]} \in C\right\}$. 
Definition 6.4. A $G_{m, n}$-partition is a set partition of $\Sigma$ into blocks such that the following conditions are satisfied:

- There exists at most one block satisfying $C^{[1]}=C$. This block will be called the zero-block.

- If $C$ appears as a block in the partition, then $C^{[1]}$ also appears in that partition.

Two blocks $C_{1}$ and $C_{2}$ will be called equivalent if there is a natural number $t \in \mathbb{N}$ such that $C_{1}=C_{2}^{[t]}$.

The number of $G_{m, n}$-partitions with $r$ non-equivalent nonzero blocks is denoted by $S_{m}(n, r)$.

For example, the following is a $G_{3,4}-$ partition:

$$
\{\{1, \overline{1}, \overline{\overline{1}}, 2, \overline{2}, \overline{\overline{2}}\},\{3, \overline{4}\},\{\overline{3}, \overline{\overline{4}}\},\{\overline{\overline{3}}, 4\}\},
$$

with a zero-block: $\{1, \overline{1}, \overline{\overline{1}}, 2, \overline{2}, \overline{\overline{2}}\}$.

We define now the concept of an ordered $G_{m, n}$-partition:

Definition 6.5. A $G_{m, n}$-partition is ordered if the set of blocks is totally ordered and the following conditions are satisfied:

- If the zero-block exists, then it appears as the first block.

- For each nonzero block $C$, the blocks $C^{[i]}$ for $1 \leqslant i \leqslant m-1$ appear consecutively right after $C$, i.e. $C, C^{[1]}, C^{[2]}, \ldots, C^{[m-1]}$.

The generalization of Theorem 1.1 in this setting is as follows.

Theorem 6.6. For all positive integers $n, m$ and $r$, we have:

$$
S_{m}(n, r)=\frac{1}{m^{r} r !} \sum_{k=0}^{r} A_{m}(n, k)\left(\begin{array}{l}
n-k \\
r-k
\end{array}\right) .
$$

The proof is similar to that of Theorem 4.1, so it is omitted.

In order to generalize Theorem 1.2, we define the falling factorial of type $G_{m, n}$ as follows: (see Equation 15 in [2])

$$
[x]_{k}^{m}:= \begin{cases}1, & k=0 \\ (x-1)(x-1-m) \cdots(x-1-(k-1) m), & 1 \leqslant k \leqslant n .\end{cases}
$$

We have:

Theorem 6.7. Let $x \in \mathbb{R}$ and $n \in \mathbb{N}$. Then we have:

$$
x^{n}=\sum_{k=0}^{n} S_{m}(n, k)[x]_{k}^{m} .
$$


We present here the idea of the proof.

Sketch of the proof. Divide the unit circle $S^{1}$ in the plane into $m$ parts according to the $m$ th roots of unity: $1, \rho_{m}, \rho_{m}^{2}, \ldots, \rho_{m}^{m-1}$, see Figure 3 , where $m=3$ and the roots are represented by small bullets. This divides the circle into $m$ arcs. Now, in each arc, locate $t$ points in equal distances from each other (see Figure 3 where $t=5$ and the points are represented by small lines). Including the point $(1,0)$, we get $x=m t+1$ points on the unit circle.

Consider now the $n$-dimensional torus $\left(S^{1}\right)^{n}=S^{1} \times \cdots \times S^{1}$ with $x^{n}$ lattice points on it. The same arguments we presented in the proof of Theorem 5.1 will apply now to Theorem 6.7 , when we interpret the $G_{m, n}$-partitions as intersections of subsets of hyperplanes in $\mathcal{G}_{m, n}$, where by $\mathcal{G}_{m, n}$ we mean the following generalized hyperplane arrangement for the colored permutations group:

$$
\begin{aligned}
\mathcal{G}_{m, n} & :=\left\{\left\{x_{i}=\rho_{m}^{k} x_{j}\right\} \mid 1 \leqslant i<j \leqslant n, 0 \leqslant k<m\right\} \\
\cup & \left\{\left\{x_{i}=0\right\} \mid 1 \leqslant i \leqslant n\right\},
\end{aligned}
$$

See e.g. [15, p. 244].

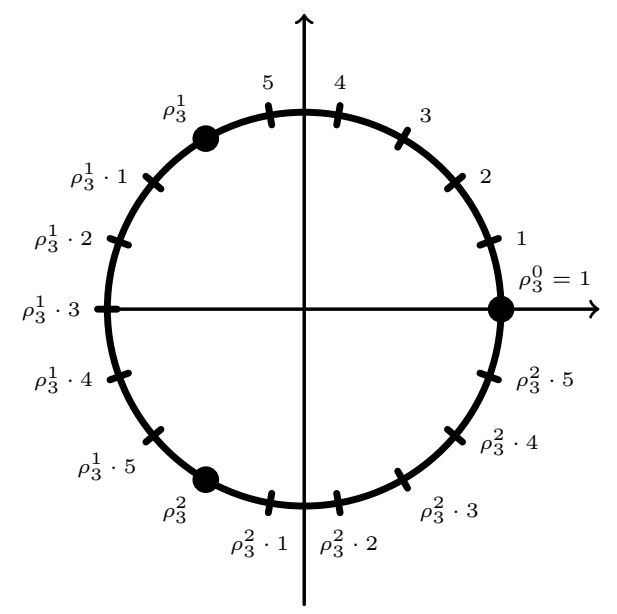

Figure 3: The 16 lattice points on $S^{1}$, representing the first coordinate for $m=3$ and $t=5$.

\subsection{The flag descent parameter for the Coxeter group of type $B$}

Another possibility to generalize these results is to consider the flag descent statistics defined on group of signed permutations. Such parameters produce, in this context, similar expressions of those presented in the previous sections, but less elegant. As an example, we show here only one of these possible extensions.

This is a different generalization of Theorem 4.1 by using the flag-descent number fdes, that is defined in [1] for a signed permutation $\beta \in B_{n}$ :

$$
\operatorname{fdes}(\beta):=2 \cdot \operatorname{des}_{A}(\beta)+\varepsilon(\beta) .
$$


where $\operatorname{des}_{A}(\beta)$ is defined as in Equation (10), and $\varepsilon(\beta)$ as in Equation (11).

We denote by $A_{B}^{*}(n, k)$ the number of permutations $\beta \in B_{n}$ satisfying $\operatorname{fdes}(\beta)=k-1$, and by $S_{B}^{*}(n, r)$ the number of $B_{n}$-partitions having exactly $r$ blocks. Here, differently from $S_{B}(n, r)$, every block counts: the zero-block is counted once, and any pair $\pm C_{i}$ is counted twice.

These two new parameters satisfy the identity stated below:

Theorem 6.8. For all nonnegative integers $n, r$ where $n \geqslant r$, we have:

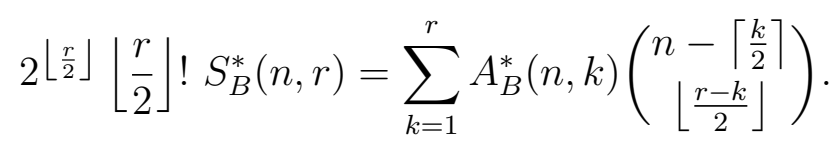

The proof uses arguments similar to those in the proof of Theorem 4.1, and is therefore omitted.

\section{Acknowledgements}

We thank an anonymous referee for his helpful comments.

\section{References}

[1] R.M. Adin and Y. Roichman, The flag major index and group actions on polynomial rings, Europ. J. Combin. 22 (2001), 431-446.

[2] P. Bala, A 3-parameter family of generalized Stirling numbers (2015). Electronic version: https://oeis.org/A143395/a143395.pdf

[3] A. Björner and F. Brenti, Combinatorics of Coxeter Groups, Graduate Texts in Math. 231, Springer-Verlag, New York, 2005.

[4] A. Björner and B. Sagan, Subspace arrangements of type $B_{n}$ and $D_{n}$, J. Alg. Combin. 5 (1996), 291-314.

[5] A. Björner and M. Wachs, Geometric constructed bases for homology of partition lattices of types A, B and D, Electron. J. Combin. 11(2) (2004), \#R3.

[6] A. Blass and B.E. Sagan, Characteristic and Ehrhart polynomials, J. Alg. Combin., 7(2) (1998), 115-126.

[7] M. Bona, Combinatorics of Permutations, Chapman \& Hall/CRC, 2004.

[8] K.N. Boyadzhiev, Close encounters with the Stirling numbers of the second kind, Math. Magazine 85(4) (2012), 252-266.

[9] F. Brenti, q-Eulerian polynomials arising from Coxeter groups, Europ. J. Combin. 15(5) (1994), 417-441.

[10] W.Y.C. Chen and D.G.L. Wang, Minimally intersecting set partitions of type B, Electron. J. Combin. 17 (2010), \#R22.

[11] W.Y.C. Chen and D.G.L. Wang, Singletons and adjacencies of set partitions of type $B$, Discrete Math. 311(6) (2011), 418-422. 
[12] L. Eulero, Methdus universalis series summandi ulterious promota. Commentarii academi æscientiarum imperialis Petropolitan æ, 8 (1736), 147-158. Reprinted in his Opera Omnia, Series 1, Vol. 14, 124-137.

[13] R. E. Knop, A note on hyper cube partitions, J. Combin. Theory (A) 15 (1973), 338-342.

[14] B.K. Miceli and J.B. Remmel, Augmented rook boards and general product formulas, Electron. J. Combin. 15 (2008), \#R85.

[15] P. Orlik and H. Terao, Arrangements of Hyperplanes, Grundleheren der mathermatische Wissenschaften 300, Springer-Verlag, Berlin-Heidelberg-New York, 1992.

[16] V. Reiner, Non-crossing partitions for classical reflection groups, Discrete Math. 177(1-3) (1997), 195-222.

[17] V. Reiner, Personal communication, 2018.

[18] J.B. Remmel and M.L. Wachs, Rook Theory, Generalized Stirling numbers and $(p, q)$-analogues, Electron. J. Combin. 11 (2004), \#R84.

[19] R.P. Stanley, An introduction to hyperplane arrangements, in: Geometric Combinatorics (E. Miller, V. Reiner, and B. Sturmfels, eds.), IAS/Park City Mathematics Series, vol. 13, American Mathematical Society, Providence, RI, 2007, 389-496.

[20] R.P. Stanley, Enumerative Combinatorics, Vol. 1, Second edition. Cambridge University Press, 2012.

[21] R. Suter, Two analogues of a classical sequence. J. Int. Seq. 3 (2000), Article 00.1.8, 18 pages.

[22] D.G.L. Wang, On colored set partitions of type $B_{n}$, Cent. Eur. J. Math. 12(9) (2014), 1372-1381.

[23] T. Zaslavsky, The geometry of root systems and signed graphs. Amer. Math. Monthly 88 (1981), 88-105. 